\title{
The Research of Graphic Features of Chinese Typeface in Application for Spreading and Popularizing
}

\author{
Fang Wu \\ Shanghai Publishing and Printing College, Shanghai, China
}

Keywords: Chinese typeface; graphic features; design application.

\begin{abstract}
Based on the study of traditional Chinese characters, this article analyzes the graphic features of Chinese Typefaces via two aspects: the structure of the stroke, and its pictographic interpretation. By understanding the graphic features of traditional Chinese characters, we can apply this knowledge to modern communication design. This article aims to reconcile the standardization of printed Chinese characters with responsibility of designers to create new, more efficient and more interesting practical field.
\end{abstract}

\section{Introduction}

There are two principal functions of typeface in Visual Identity design, conveying the information and showing the image. Examples of good typeface design have both.

Because of the literal meanings, typeface is not only the graphic element of Visual Identity design but it is constructive and crucial. Currently, with Chinese design on the threshold of advancing from "Made in China" to "Design in China", Chinese communication design will inevitably face difficulties from the different nation, culture, language, religion and other aspects of intercultural communication. How to take advantage of the pictographic features of Chinese characters is the key to finally conquering the Babel predicament. Pictography will help typeface design become more international, while visualization can express the meaning more efficiently. For example, the emblem of 2008 Beijing Olympic Game is based on the image of a Chinese character. (Fig.1) The design concept takes advantage of the similarity between traditional Chinese character and pattern association. It uses the Chinese character "jing", which represents Beijing as the main image. (Fig.2) Meanwhile, this character image also describes a dancing figure. People without any knowledge of Chinese language can perceive the meaning without words. The success of this emblem design seems to testify that there is a common visual language. And this common language combines typeface structure with graphic composition, enabling people to understand the meaning in a very efficient way. Looking into the graphic features of traditional Chinese typeface design can help us to better understand the different modes of Chinese characters' visualization. Moreover, it may also provide some inspiration for Chinese design in the future.

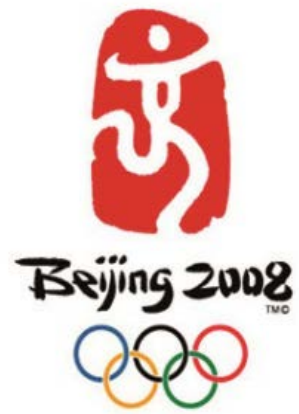

Fig.1 Emblem of 2008 Beijing Olympic Game

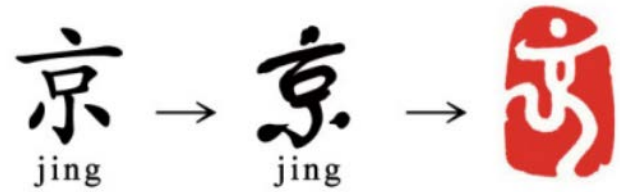

Fig.2 change from the character to image

The Present Situation and Research Value of Chinese Typeface. Initially speaking, there's no 
essential difference in creation between traditional art and modern art. But through all the society and times change, traditional art differs gradually from palace art and commercial art. Especially for the traditional typeface design which always focuses on visible, immediate, graphic, symbolic feature, has become more and more researchable by its distinctive way of creativity. Unfortunately, this research has been largely neglected by local Chinese designers. There are two main reasons for the loss of traditional Chinese typeface study. First, the standardized printing has made Chinese printing fonts less graphic and symbolic. Thus, traditional typeface seems not applicable to the modern printing industry. Second, unlike the alphabetic writing of English, Chinese belongs to ideographic writing, which means Chinese typeface cannot be presented as a line composition. Chinese characters in modern printing do not connect with each other. They are separated, individual, and abstract. With the loss of pictographic and graphic features, Chinese characters are becoming much more difficult to understand. Therefore, graphic designers may regard Chinese characters as potential obstacles to intercultural communication, causing a lost interest in Chinese typeface design.

The study of the graphic nature of Chinese typeface can benefit the national cultural transmission of graphic design in the following three aspects:

Identification. Living in the information age, visual identity systems are responsible for information exchange: how people recognize and remember the information is the first question for all the designers. Distinctive features can leave a strong impression on target audiences. Originality can make uniquely special. Localization can promote the awareness of various nations and regions. Through traditional Chinese typeface, we can get these three - Distinctive features, originality and localization - to make information easy to identify. Usually, words and graphics are two important elements in Visual Identity design. If traditional Chinese typeface is combining characters' structures with symbolic graphics, there should be something necessary to study and conclude from this art in local folklore.

Communication. Chinese typeface has different functions in art and in VI design. In most art work, especially modern art, traditional Chinese writing is adopted as some spiritual symbol. For example, when the calligraphy elements in the art work like cursive script, the modality is more important than the meaning itself. Artists want audiences to feel instead of reading. However, in the field of communication design, feeling some kind of spirit is far from spreading the effective information. And readability is essential. In this case, knowing the possibility of graphic representation in Chinese typeface design is vitally important. The graphic feature can lower the understanding obstacles in communication.

Diversification. Art and culture exist in diversity which includes different nationalities, species, linguistics, etc. Diversification can lead to recognition and identification. For the study of traditional Chinese typeface, it's also the rediscovery of native culture. The study will help local designers get the confidence of brand-new typeface design. And comparing with the reference and imitation, the aim we are looking for is the innovation, or even more, evolution. To transform cultural symbols from traditional typeface into modern design is not only an opportunity for local people to reacquaint, but it's an opportunity for old tradition to be reborn. And with the stimulation of this renascence, people from other places may find the chance to get closer to real Chinese culture.

\section{The Principle of Two Graphic Ways in Chinese Typeface}

\subsection{From Visual Images to the Structures of Typeface.}

Both oracle bone inscriptions and inscriptions on ancient bronze objects of China are based on actual visual images. At the very beginning of Chinese typeface, the structure of the image is simply the strokes of character. The Chinese pictography is generally comprised of four different types of image sources, namely, elements of the human body, the front or side of animals, natural symbols, and artificial objects and symbols (Fig.3). We find numerous examples of the deep connection between images and stroke structure. This feature makes the meaning of traditional Chinese characters much easier to recognize. Although through the years, the characters have become more 
and more concise, people can still grasp the initial idea of this graphic feature.

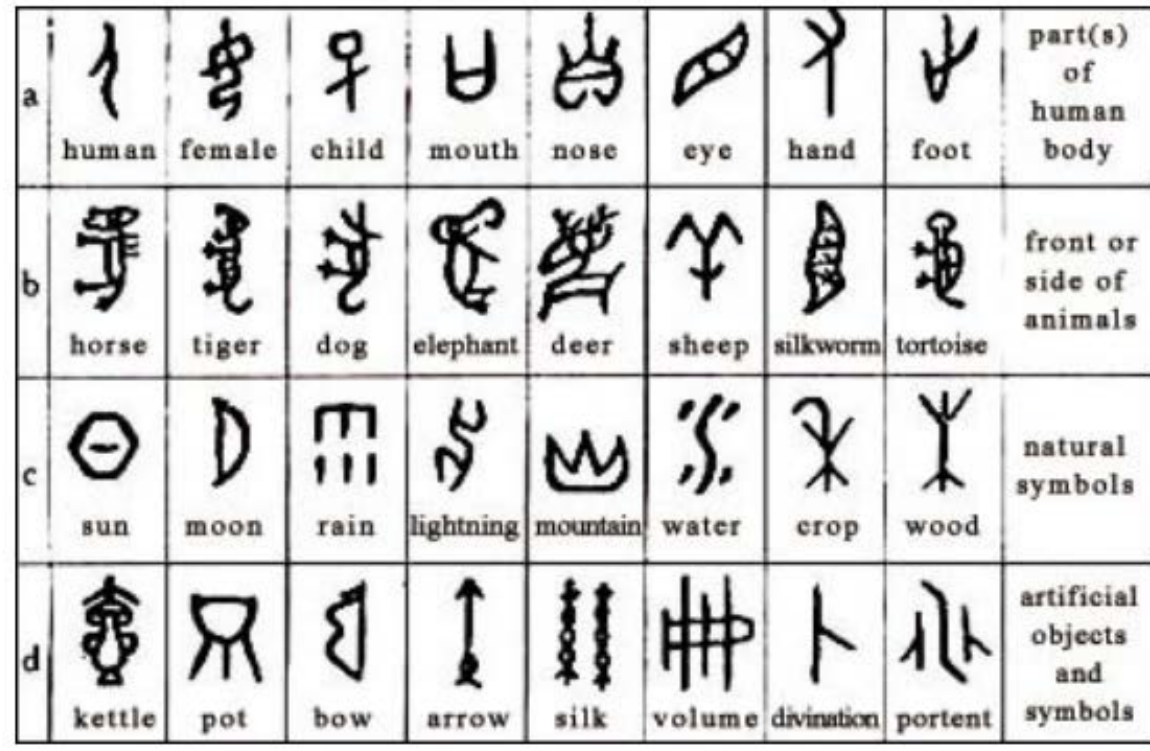

Fig.3 four kinds of image sources for pictograph

In the field of Chinese pictograph, the characters can be divided into two groups, single image and collective images. They both obey the graphic rules of pictograph, but show different compositions of strokes.

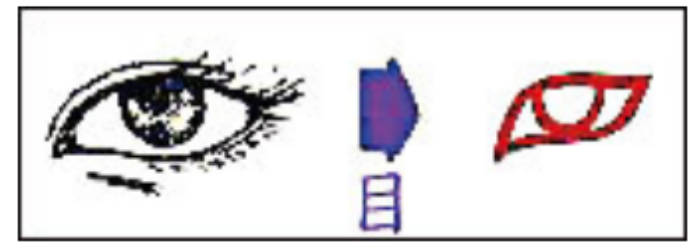

Fig.4 Chinese character "Eye"

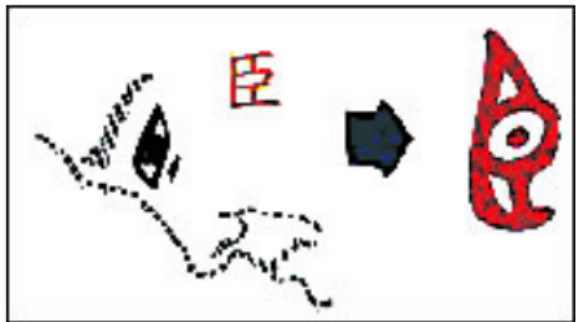

Fig.5 Chinese character "Minister"

Fig. 4 describes how the single image of a human eye becomes the Chinese character "eye". The right red symbol is the traditional character, while the purple one is the modern symbol. It's clear that the traditional character represents the original single image so directly that everyone could understand the meaning easily. Fig.5 shows the development of the character "minister". It's interesting to notice that the traditional character "minister" on the right is perpendicular to the traditional character "eye". That is because in ancient China, the minister would always stoop to obey the emperor's command. This is the reason why ministers' eyes are represented as perpendicular. The different angles of the character reveal the different class and identity of people. It's interesting to know the society and culture through traditional characters. Unfortunately, this wonderful detail of Chinese character has been lost in the modern style because the upright "eye" is much more suitable for printing.

Chinese pictograph with single images can not only indicate noun but also a verb. Fig.6 indicates the lively formation of character "cross". The character was created by the inspiration of the cross position of human legs.

Besides the single image, Chinese pictographs frequently have collective images. Fig.7 indicates the character "brow". Compared to the character "eye", character "brow" combines two images in the construction. The combination shows the meaning of the character, and also fixes the position of the brow. Moreover, a Chinese pictograph is usually formulated from two or more than two single image characters. As shown in Fig.8, the left character means "human" with a single image of the human body, while the right character means "follow" with two images of the human body. The creating of 
characters here is like a simple addition, simple but not boring. The impressive part of this character's formulation is that the combination of two images expresses exactly what "follow" means: one person after another, two nouns make a verb.

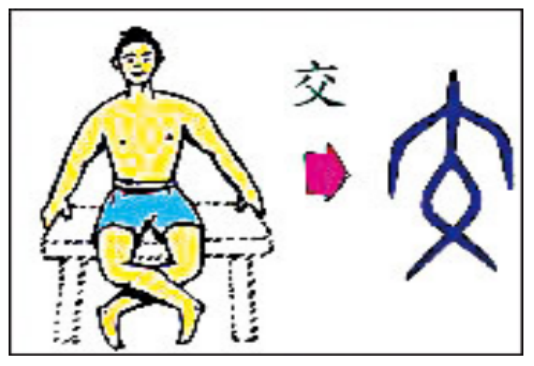

Fig.6 Chinese character "Cross"

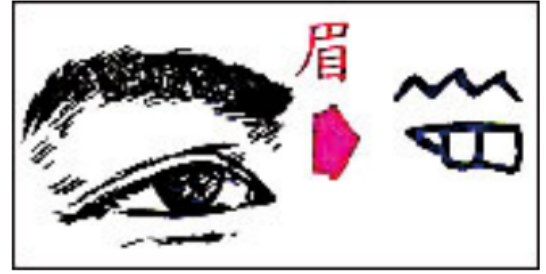

Fig.7 Chinese character "Brow"

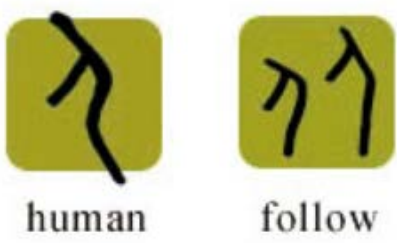

Fig.8 single image and collective images

\subsection{From Structures of Typeface to Creative images.}

In most forms of pictography, images exist for the characters. That means the content of the image depends on the meaning of the character. And once people obtain the ability of reading, it's easy to pay more attention to the literal meanings of both characters and images but somehow, to forget that the structures of characters are also the elements for making images. For some art skills of traditional Chinese typeface which are actually much closer to design, strokes and structures are available for creating new images. The shape of images still depends on the structure of the character, but graphic creation beyond the literal meaning becomes the purpose of the character.

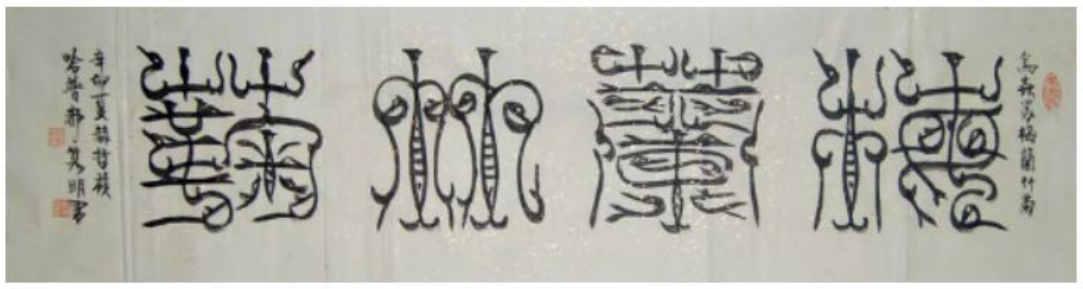

Fig.9 Chinese seal characters with birds and insects

From right to left: plum blossom, orchid, bamboo and chrysanthemum

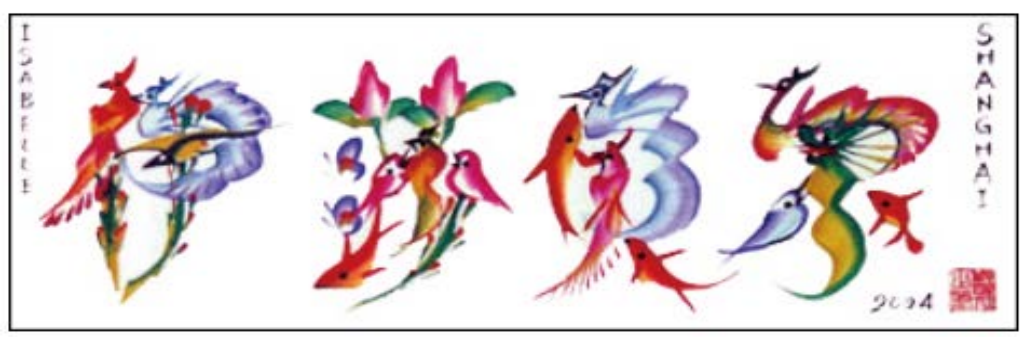

Fig.10 Chinese folk characters with flowers and birds

In the Annals of Spring and Autumn (from 770 to 221 B.C.), ancient Chinese started to decorate the seal characters with different shapes of birds and insects. The birds and the insects' images were developed from the strokes of the seal character without necessarily implying connections (Fig. 9). This is the beginning of another graphic style of Chinese typeface. This decoration method can also be found in many Chinese folk typefaces. As shown in Fig.10, the characters are decorated with vivid 
flowers and birds. Actually these four characters translate "Isabelle" into Chinese. As we know, here images do not have to represent the meaning of characters. This kind of graphic feature in Chinese typeface leaves much more spaces for images' creation. Moreover, it's also the inspiration for modern Chinese design. Although graphic features of Chinese typeface are fading away in the practical use, decoration with images will be the breakthrough of innovation for Chinese characters.

If modern Chinese typeface design could borrow this feature, it can make the work unique and interesting. Considering the importance of effective information in modern design, it would be more meaningful if typeface design included some representative images. In Fig.11, the representative images are not only developed from the strokes, but replace the strokes. The four characters each indicate "fall", "spring", "winter" and "summer". A creative designer picks typical images to describe the characters. The characters are no longer entirely formulated as images as in the pictography but the partial image details make the idea fresh and modern.

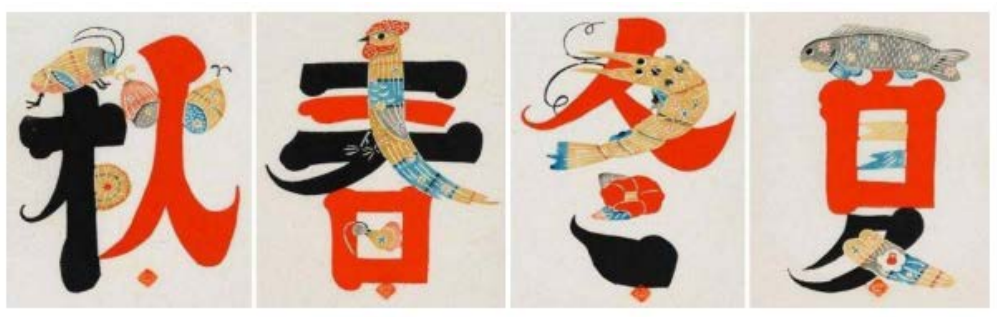

Fig.11 Chinese characters with representative images

\section{Applications of the Graphic Feature in New Typeface Design}

\subsection{Image Describes the Character.}

Images and characters share one function: the correct transmission of information. Only if the image is formed by the structure of character with exactly the same meaning, will the transmission be more efficient and correct. Using the graphic feature to combine image and character can help modern typeface design both visually and literally.

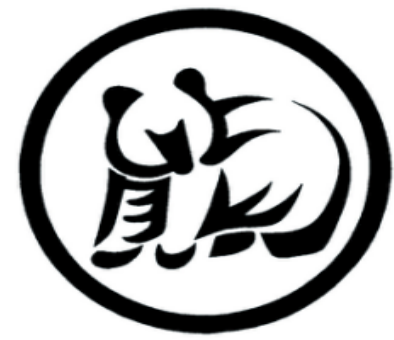

熊

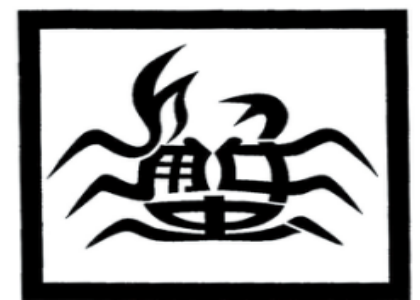

蟹

Fig.12 Chinese character "Bear"

Fig.13 Chinese character "Crab"

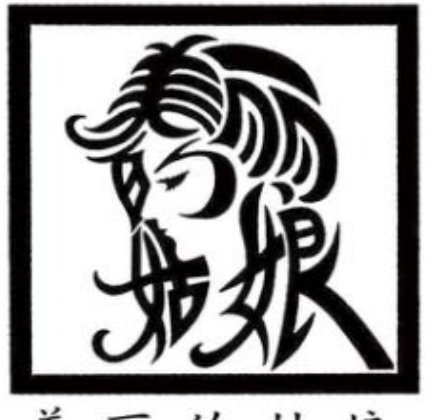

$$
\text { 美 丽的 姑 娘 }
$$

Fig.14 Chinese characters "Nice Girl"

As shown in Fig.12 and 13, typeface design attempts to express the character meanings by forming images. Comparing the standard printed Chinese characters, the images almost follow all the 
structures of the characters. In this group of examples, image, character and meaning are "three in one". Chinese language is a typical isolating language, so one character could indicate the full meaning. Sometimes with this idea of design, designers also put different characters together to make a phrase or short sentence. Fig.14 includes five Chinese characters to make a phrase which means "nice girl". These five characters are also the indispensable elements of the girl's image. By using the graphic feature of characters, modern typeface design expands the potential advantages of text and graphics.

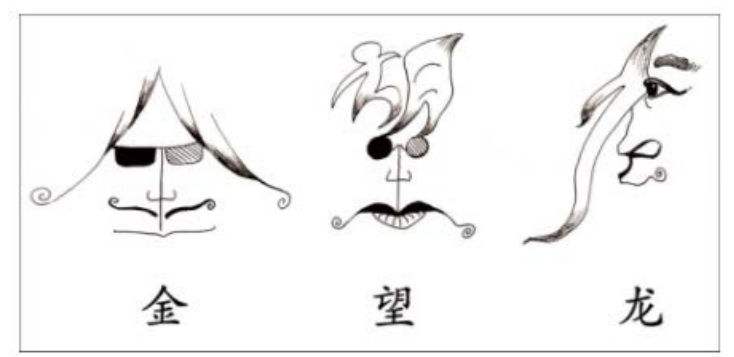

Fig.15 student's work: new typeface design of name

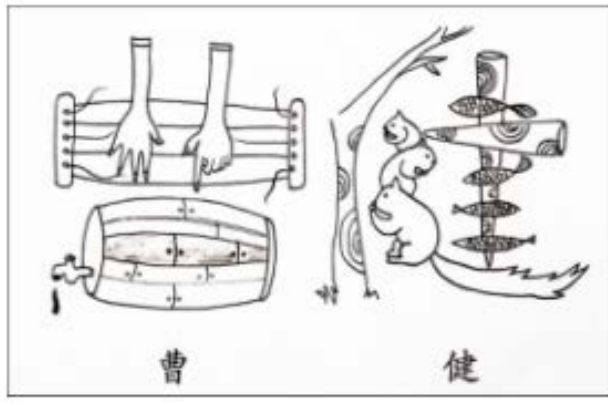

Fig.16 student's work: new typeface design of name

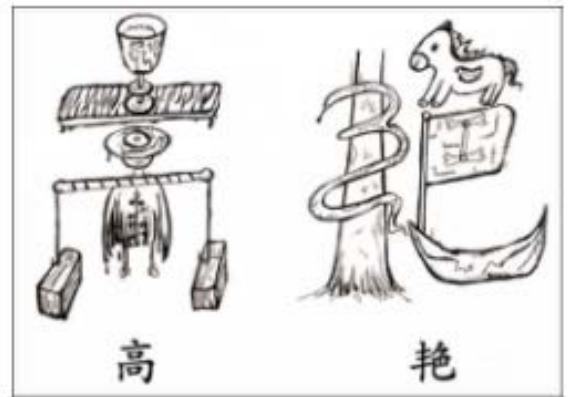

Fig.17 student's work: new typeface design of name

\subsection{Image Decorates the Character.}

In this way of modern Chinese typeface design, structures and strokes are the essential layout for developing images. To some extent, images are the transformation from characters. Figs.15 to 17 show three examples of students' work. They try to re-design their name characters as graphics. As shown in Fig.15, corresponding to the standard Chinese characters, the images fit perfectly. And the topic of this new typeface work is "Pirate" which has nothing to do with the meaning of the characters. Graphic feature in this section is absolutely based on the strokes of standard characters. Figs.16 and 17 use a variety of dramatic images to form the characters' structure of the first name and second name. All the images finally create the scene for some story. This method of decoration makes more free creative space for typeface designers by limiting the restriction of the literal meanings when necessary.

Standard printed Chinese characters are in the shape of square. The regular, limited spaces may eliminate the opportunity for change and innovation. Although it can not always be ascribed to the objective situation, decorating strokes with images do make the typeface more attractive. Moreover, this graphic way of Chinese typeface design can provide the additional function which regards characters as not only abstract symbols, but basic elements of the image.

\section{Conclusion}

Any development of art and culture has some inevitable reason through ages. During its evolution from traditional pictography to modern standard characters, Chinese typeface has changed its written directionality, writing tools, external forms, and even the original graphic creativity. Although we cannot reverse this evolution, there is a compelling need to separate Chinese typeface design from standard printing fonts. The truth is they have different targets. Therefore, supporting the standardization of the printing industry doesn't mean we must abandon the creative passion for 
Chinese typeface. In the future, the graphic features of characters will be one of the highlights of modern Chinese design. Designers should take the responsibility of creating new, more efficient and more interesting Chinese typefaces to survive in this vast information world.

\section{Acknowledgement}

Education and Scientific Research Project of Shanghai Municipal Education Commission (C17071). Education resource database Project of Shanghai Publishing and Printing College.

\section{References}

[1] LU Sheng-zhong. Goodbye to the Tradition 4M. Beijing: Joint Publishing Company, 2004.

[2] WANG Hai-xia. Perspective: The Folk Art of Chinese Folk CultureM. Xian: Taibai Literature and Art Publishing House, 2006.

[3] LIU Hua-di. Expression of the Elements Features of Graphic Language Imaginary in the Modern Advertising DesignJ. Packaging Engineering, 2013, 34(6):5-7.

[4] XU Yang. Visual Identity DesignM. Beijing: China Ethnic Photographic Art Publishing House, 2011.

[5] CUI Sheng-guo. Shape Expressing: Thinking about the Visual Form and Meaning of the GraphicsJ. ZhuangShi, 2013, 02: 127-128.

[6] ZHANG Dao-yi. How Beautiful the Chinese Characters areM. Shanghai: Shanghai Brilliant Publishing House, 2012. 